\title{
$\begin{array}{ll}\text { Research Square } & \text { Preprints are preliminary reports that have not undergone peer review. } \\ \text { They should not be considered conclusive, used to inform clinical practice, }\end{array}$
}

\section{Hybrid Optimization-Based Robust Watermarking Using Denoising Convolutional Neural Network}

Dhiran Kumar Mahto

NIT Patna: National Institute of Technology Patna

Amit Singh ( $\square$ amit.singh@nitp.ac.in )

NIT Patna: National Institute of Technology Patna

\section{Research Article}

Keywords: Copyright protection, Optimization, Security, Watermarking, Transform domain, DnCNN

Posted Date: November 16th, 2021

DOI: https://doi.org/10.21203/rs.3.rs-1055534/v1

License: (c) (1) This work is licensed under a Creative Commons Attribution 4.0 International License. Read Full License 


\section{Abstract}

Colour images have been widely used in many aspects of life; however, copyright violation issues related to these images motivate research efforts. This paper aims to develop an enhanced watermarking algorithm for producing a watermarked image using hybrid optimisation with high imperceptibility and robustness. The algorithm is based on spatial and transform domains and begins by embedding multiple secret marks into cover media using an optimal scaling factor. The multi-type mark contributes an additional level of authenticity to the proposed algorithm. Furthermore, the marked image is encrypted using an improved encryption scheme, and the denoising convolutional neural network (DnCNN) is employed to enhance the robustness of the proposed algorithm. The results reveal that the proposed watermarking algorithm yields low computational overhead, excellent watermark capacity, imperceptibility, and robustness to common filtering attacks. Moreover, the comparison shows that the proposed algorithm outperforms other competing methods.

\section{Introduction}

In recent years, internet-based services have been widely used, and colour images are extensively shared online as a common information carrier [1]. Online services such as social media, e-banking, e-healthcare and e-learning have become an important part of our day-to-day activities [2]. This insecure channel has been effectively used for the last few decades for the transmission of multimedia content. Internet technologies have proved to be high speed, indispensable and cost-effective at transmitting media, but, at the same time, they do not provide reliable security when transmitting such content [3]. In these circumstances, transmitting media can be risky and, due to certain security concerns, is an open issue for potential researchers to examine how to make multimedia content more secure. In general, to address these problems, robust and secure watermarking schemes have drawn the attention of the scientific community. In this scheme, digital marks are invisibly concealed into carrier media to maintain the ownership and integrity of multimedia content $[4,5]$. A digital mark (s) is a data string that can be used for several purposes. Digital watermarking properties such as robustness, invisibility and embedding capacity are mutually exclusive in nature and, therefore, must be balanced to achieve high performance [6].

Optimisation-based watermarking is frequently used by researchers to manage invisibility and robustness at the same time [7-10]; however, these approaches still suffer from limited watermark capacity and security.

To overcome the issues discussed above, we have developed an enhanced watermarking algorithm for colour images. Our contributions are summarised as follows:

1) The fusion of spatial (magic cube) and transform domain (LWT-Schur-T-SVD ) encryption methods are adopted to implement our algorithm, which embeds multiple marks. LWT examines the input in integer form and eliminates the reversibility issue of other wavelet transforms [11]. Furthermore, Schur decomposition allows for speedier and more robust watermarking [12]. The tensor SVD [13], as opposed to the traditional SVD, is used to embed watermarks for improved robustness, and a pseudo-magic cube scheme [14] is used to enhance embedding capacity.

2) Hybrid optimisation procedures called 'HPSOF' [15] have been designed and are employed to compute the scaling factor. A good relationship between invisibility versus robustness is maintained through the factor.

3) To enhance security, improved SIE scrambling [16] is used to encrypt the watermark image.

4) DnCNN is used on the recovered watermark image to improve the scheme's robustness [17].

5) Multiple marks are concealed in the host media channel to produce the final watermark. This contributes an additional authenticity to the suggested algorithm. 
The remaining sections of this paper are arranged as follows: the literature review is presented in section 2; the proposed hybrid optimisation-based watermarking approach is discussed in section 3; the experimental results are presented in section 4; and the paper concludes in Section 5.

\section{Related Work}

In this section, a few well-known related colour image watermarking techniques are briefly discussed, and Table 1 summarises and compares the contributions of various notable methods. Sharma et al. [18] demonstrated a watermarking approach using artificial intelligence in the transform domain. To provide an additional level of security, the mark is encrypted with chaotic maps. To embed the mark, the singular score of the host media is modified with respective RGB channels of principle components of encrypted watermarks. Experiments demonstrate that the scheme is robust and secure. In [19], the author suggested spatial domain-based watermarking using Schur decomposition. This scheme has the advantages of spatial as well as transforms domain techniques, resulting in fewer computations and improved robustness; however, it has a lower embedding capacity. Sharma et al. [20] demonstrated a watermarking approach incorporating the fusion of lifting wavelet transform (LWT) and discrete cosine transform (DCT) techniques and further applied the artificial bee colony (ABC) algorithm to improve visual quality and robustness. This scheme is robust against various attacks, but a detailed security analysis needs to be conducted. Singh [21] developed a robust watermarking scheme for telehealth applications using LWT and DCT techniques. Before being concealed in the host media, the signature watermark is encrypted with message-digest (MD5) coding, and the patient report is encoded with $\mathrm{BCH}$ coding to enhance the robustness and privacy of the scheme; however, the scheme is less robust following a few attacks. Mohan et al. [22] developed a hybrid optimisation-based watermarking approach using an optimisation algorithm that enhances quality and robustness. Here, an additional level of security is provided by applying selective encryption to the host media at a low cost. In [23], the author implemented a spatial domain-based scheme using encryption and direct current coefficients. The mark is divided into different sub-watermarks and is then encrypted using MD5. Furthermore, the encrypted watermarks are concealed in the blue channel components. The presented scheme has improved robustness and the analysis ensures invisibility; however, the embedding capacity needs to be improved. Zear and Singh [24] suggested a hybrid watermarking scheme based on LWT-DCT-SVD to secure multimedia content. The security of the marked image and robustness of the text mark are improved by employing MD5 and Hamming errorcorrecting codes, respectively. The present technique is lacking in terms of BER and optimisation. Kumar and Singh [25] presented a colour image watermark in $\mathrm{YCbCr}$ where the embedding and extraction are processed through an alpha blending scheme in the LWT domain. Here, security is enhanced via Arnold's cat map (ACM). This scheme needs to be improved against median filter attacks. Loan et al. [26] designed a watermarking method that is applicable to both grey and colour images using DCT. The present technique uses a double layer of security by employing chaotic encryption and ACM for watermarked images. From the experimental results, it is noted that the method provides better performance in terms of security, quality and robustness; however, the complexity and cost are high. Haghighi et al. [27] implemented LWT, DCT and a feed-forward neural network-based semi-fragile scheme for temper detection and recovery. Using the inverse halftoning technique, the tempered regions are identified in the recovery stage. 
Table 1

Summary of some related approaches

\begin{tabular}{|c|c|c|c|c|}
\hline Scheme & Technique used & Domain & $\begin{array}{l}\text { Color } \\
\text { model }\end{array}$ & Limitations \\
\hline $\begin{array}{l}\text { Sharma et al. } \\
\text { [18] scheme }\end{array}$ & $\begin{array}{l}\text { Chaotic map, ABC optimization, } \\
\text { DWT, SVD }\end{array}$ & transform & RGB & $\begin{array}{l}\text { Tested with only single type } \\
\text { watermark }\end{array}$ \\
\hline $\begin{array}{l}\text { SU et al. [19] } \\
\text { scheme }\end{array}$ & Schur, Arnold transforms & Spatial & RGB & Lower Embedding capacity. \\
\hline $\begin{array}{l}\text { Sharma et al. } \\
\text { [20] scheme }\end{array}$ & $\begin{array}{l}\text { LWT, DCT, ABC optimization, } \\
\text { Arnold transforms }\end{array}$ & transform & RGB & $\begin{array}{l}\text { Watermark Security analysis not } \\
\text { confirmed }\end{array}$ \\
\hline $\begin{array}{l}\text { Singh [21] } \\
\text { scheme }\end{array}$ & $\begin{array}{l}\text { LWT, DCT, message-digest } \\
\text { (MD5) }\end{array}$ & transform & YIQ & $\begin{array}{l}\text { Less robust against rotation and } \\
\text { Histogram Equalization }\end{array}$ \\
\hline $\begin{array}{l}\text { Mohan et al. [22] } \\
\text { scheme }\end{array}$ & $\begin{array}{l}\text { DWT, SVD, HD, step space-filling } \\
\text { curve, HPSOF }\end{array}$ & transform & YIQ & $\begin{array}{l}\text { Computational analysis not } \\
\text { confirmed }\end{array}$ \\
\hline $\begin{array}{l}\text { Su and chen [23] } \\
\text { scheme }\end{array}$ & DC coefficients & Spatial & RGB & Lower embedding capacity \\
\hline $\begin{array}{l}\text { Zear and singh } \\
\text { [24] } \\
\text { scheme }\end{array}$ & $\begin{array}{l}\text { LWT, DCT, SVD Hamming Error } \\
\text { Encoder, }\end{array}$ & transform & $\begin{array}{l}\text { RGB, } \\
\text { YIQ, } \\
\text { YCbCr }\end{array}$ & $\begin{array}{l}\text { scaling factor could be determined } \\
\text { through optimization. }\end{array}$ \\
\hline $\begin{array}{l}\text { Kumar and singh } \\
\text { [25] } \\
\text { scheme }\end{array}$ & $\begin{array}{l}\text { LWT, alpha blending, Arnold cat } \\
\text { map }\end{array}$ & transform & $\mathrm{YCbCr}$ & $\begin{array}{l}\text { Less robust against Median- } \\
\text { filtering attack }\end{array}$ \\
\hline $\begin{array}{l}\text { Loan et al. [26] } \\
\text { scheme }\end{array}$ & $\begin{array}{l}\text { DCT, Arnold transforms, chaotic } \\
\text { encryption }\end{array}$ & transform & $\mathrm{YCbCr}$ & High complexity and computation. \\
\hline $\begin{array}{l}\text { Haghighi et al. } \\
\text { [27] scheme }\end{array}$ & $\begin{array}{l}\text { LWT, DCT, feed-forward neural } \\
\text { network, halftoning }\end{array}$ & transform & YUV & $\begin{array}{l}\text { false-positive rates for } \\
\text { compression attack and less } \\
\text { robust against average and } \\
\text { median filters }\end{array}$ \\
\hline
\end{tabular}

\section{Proposed Algorithm}

In this section, the proposed algorithm, including computation of the embedding factor, the embedding and extraction phases of multiple marks, and the denoising process of recovered mark data, are described in detail. Figure 1 shows the block diagram of the proposed approach.

\subsection{Watermark embedding and extraction procedure}

In the embedding process, the colour host image, 'cover_img', is initially converted into red, green and blue components represented by 'Rc', 'Bc' and 'Gc', respectively.

This algorithm subsequently utilises DWT to decompose the 'Rc' component into a different sub-band and conceals the PAN number, 'PAN', in the 'HHr' sub-band of the image. Next, the account number, 'Account', is concealed in the green 
channel, 'Gc', using the magic cube, 'm_cube', algorithm. Following this, the watermark image, 'w_image', is scrambled using the improved SIE scheme and is followed by the T-SVD decomposition. The blue component, 'Bc', is decomposed using the fusion of LWT, Schur and T-SVD. The resultant singular matrix, 'Sb', is altered using the scrambled watermark, 'enc_wimg', and the optimised scaling factor value, 'opt_a'. Lastly, the final watermarked image is formed by combining all three marked channels (wat_R, wat_G, wat_B). The watermark extraction procedure is the inverse of the embedding process. The complete embedding and extraction processes are explained in Algorithm 1 and Algorithm 2, respectively. Table 2 lists the specifics of the notations used in the algorithms.

Table2. Description of the notations used in the algorithms 


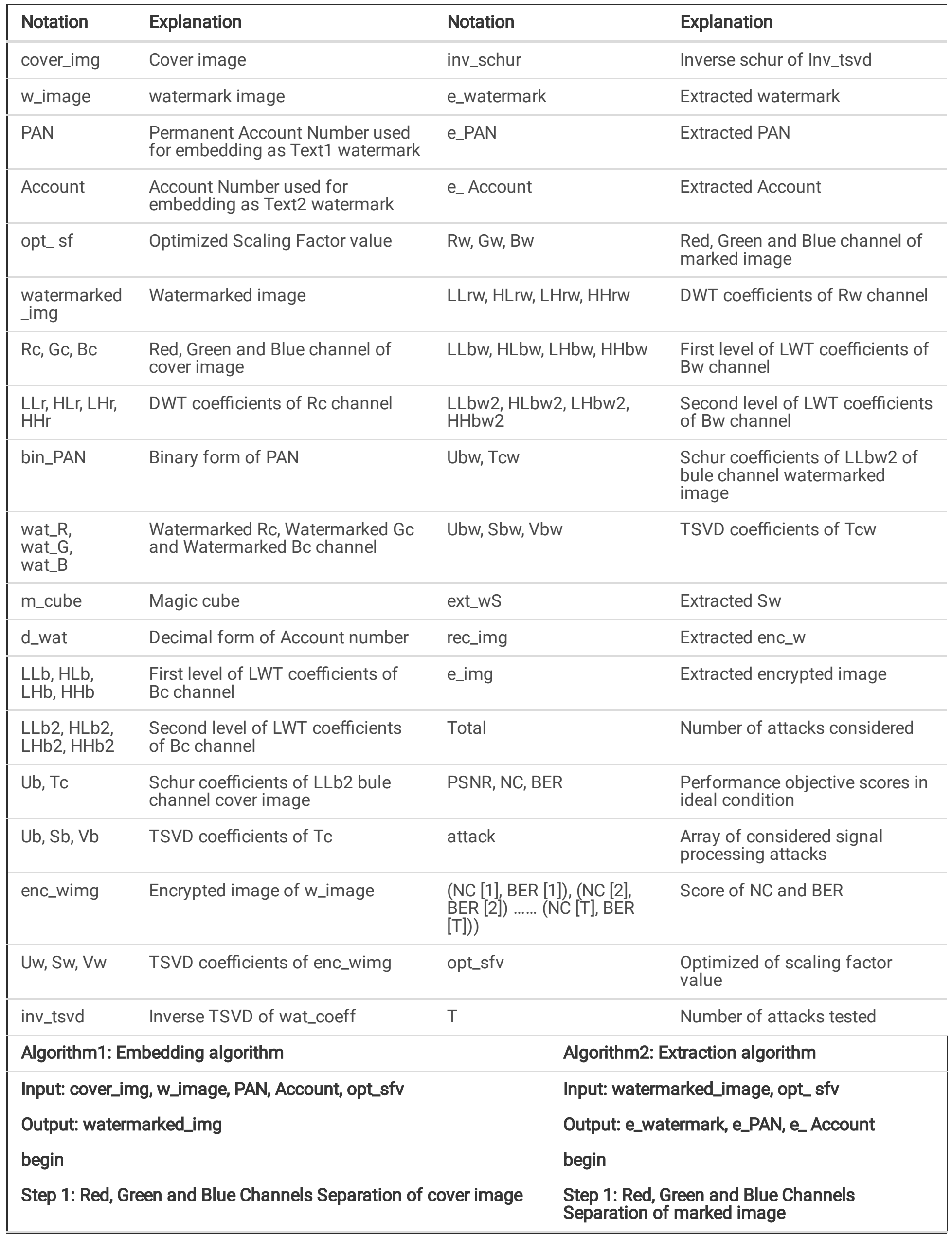




\begin{tabular}{|c|c|c|}
\hline Explanation & Notation & Explanation \\
\hline \multicolumn{2}{|l|}{ 1. Rc $\leftarrow$ cover_img $(:,:, 1)$} & 1. Rw $\leftarrow$ watermarked_img $(:,:, 1)$ \\
\hline \multicolumn{2}{|l|}{ 2. Gc $\leftarrow$ cover_img $(:,:, 2)$} & 2. Gw $\leftarrow$ watermarked_img $(:,,, 2)$; \\
\hline \multirow{2}{*}{\multicolumn{2}{|c|}{$\begin{array}{l}\text { 3. Bc } \leftarrow \text { cover_img }(:,:, 3) \\
\text { Step 2: Embedding in Red channel }\end{array}$}} & 3. Bw $\leftarrow$ watermarked_img $(:,:, 3)$; \\
\hline & & Step 2: Extraction from Red channel \\
\hline \multicolumn{2}{|l|}{ 4. [LLr, HLr, LHr, HHr] $\leftarrow$ DWT (Rc, 'Haar'); } & 4. [LLrw, HLrw, LHrw, HHrw] $\leftarrow$ DWT (Rw, 'Haar'); \\
\hline \multicolumn{2}{|l|}{ 5. bin_PAN $\leftarrow$ Binary $(P A N)$} & 5. e_PAN $\leftarrow$ Text_Extract $(\mathrm{HHrw}$, len); \\
\hline \multicolumn{2}{|l|}{ 6. for $\mathrm{i}=1$ : Length (bin_PAN) do } & Step 3: Extraction from Green channel \\
\hline \multicolumn{2}{|l|}{ 7. if bin_PAN [i] = = 0} & $\begin{array}{l}\text { 6. e__ Account } \leftarrow \text { extract_ MagicCube }(\mathrm{Gw}, \mathrm{sz} \text {, } \\
\text { NN, seed, rx, ry, rz); }\end{array}$ \\
\hline \multicolumn{2}{|l|}{$\begin{array}{l}\text { 8. bin_PAN }[i] \leftarrow-1 \text {; } \\
\text { 9. end if }\end{array}$} & 7. $e_{-}$Account $\leftarrow$ Decimal 2 Char $\left(e_{-}\right.$Account $)$; \\
\hline \multicolumn{2}{|l|}{ 10. end for } & Step 4: Extraction from Blue channel \\
\hline \multicolumn{2}{|l|}{ 11. len $\leftarrow$ Length (bin_PAN); } & $\begin{array}{l}\text { 8. [LLbw, HLbw, LHbw, HHbw] } \leftarrow \text { LWT }(B w \text {, } \\
\text { 'Haar'); }\end{array}$ \\
\hline \multirow{2}{*}{\multicolumn{2}{|c|}{$\begin{array}{l}\text { 12. wat_HHr } \leftarrow \text { Embed }(\mathrm{HHr} \text {, bin_PAN, opt_sfv, len); } \\
\text { 13. wat_R } \leftarrow \text { IDWT }(\mathrm{LLr}, \mathrm{HLr}, \mathrm{LHr} \text {, wat_HHr, 'Haar'); }\end{array}$}} & $\begin{array}{l}\text { 9. [LLbw2, HLbw2, LHbw2, HHbw2] } \leftarrow \text { LWT } \\
\text { (LLbw 'Haar'); }\end{array}$ \\
\hline & & 10. $[\mathrm{Ubw}, \mathrm{Tcw}]=$ schur (LLbw2). \\
\hline \multicolumn{2}{|l|}{ Step 3: Embedding in Green channel } & 11. [Ubw, Sbw, Vbw] $\leftarrow$ TSVD $(T c w)$; \\
\hline \multicolumn{2}{|l|}{ 14. d_wat $\leftarrow$ Char2Decimal (Account); } & 12. ext_wS $\leftarrow($ Sbw $-S w) /$ opt_sfv; \\
\hline \multicolumn{2}{|l|}{$\begin{array}{l}\text { 16. wat_G } \leftarrow \text { Text_Embedding }\left(\mathrm{Gc}, \mathrm{d} \_w a t, \mathrm{~m} \_c u b e\right) \text {; } \\
\text { Step 4: Embedding in Blue channel }\end{array}$} & $\begin{array}{l}\text { 13. rec_img } \leftarrow \text { Uw } \times \text { ext_wS } \times \text { Vw; } \\
\text { 14. e_img } \leftarrow \text { decrypt (rec_img); }\end{array}$ \\
\hline \multicolumn{2}{|l|}{ 17. [LLb, HLb, LHb, HHb] $\leftarrow$ LWT $(B c$, 'Haar'); } & 15. e_watermark $\leftarrow$ DnCNN (e_img); \\
\hline \multicolumn{3}{|l|}{ 18. [LLb2, HLb2, LHb2, HHb2] $\leftarrow$ LWT (LLb, 'Haar'); } \\
\hline \multicolumn{3}{|l|}{ 19. $[\mathrm{Ub}, \mathrm{Tc}]=$ schur(LLb2); } \\
\hline \multicolumn{3}{|l|}{ 20. [Ub, Sb, Vb] $\leftarrow$ TSVD $(T c)$; } \\
\hline \multicolumn{3}{|l|}{ 21. enc_wimg $\leftarrow$ encrypt (w_image); } \\
\hline \multicolumn{3}{|l|}{ 22. [Uw, Sw, Vw] $\leftarrow$ TSVD (enc_wimg); } \\
\hline \multicolumn{3}{|l|}{ 23. wat_embd $\leftarrow S b+$ opt_sfv $\times$ Sw; } \\
\hline \multicolumn{3}{|l|}{ 24. wat_coeff $\leftarrow \mathrm{Ub} \times$ wat_embd $\times \mathrm{Vb}$; } \\
\hline \multicolumn{3}{|l|}{ 25. inv_tsvd $\leftarrow$ iTSVD (wat_coeff); } \\
\hline \multicolumn{3}{|l|}{ 26. inv_schur $\leftarrow$ i schur (Inv_tsvd); } \\
\hline \multicolumn{3}{|l|}{ 27. wat_B $\leftarrow$ i LWT(Inv_schur); } \\
\hline 28. watermarked_img $\leftarrow$ combine $($ wat_R, wat_G, w & & \\
\hline return watermarked_img & & \\
\hline
\end{tabular}




\subsection{Determination of optimal embedding factor value}

To determine the ideal optimal embedding factor value for the embedding of multiple watermarks, the HPSOF optimisation scheme is used, which provides a well-balanced trade-off between invisibility and robustness. PSO and Firefly are swarm intelligence-based metaheuristic methods inspired by nature. To minimise the cost of complex numerical problems, Aydilek used a fusion of PSO and Firefly [15] optimisation techniques. Table 3 includes the parameters used for controlling the algorithms to produce optimal outcomes. Also, the fitness value objective function is defined as:

$$
F_{v}=\left\lfloor\gamma \times\left(\frac{1}{P S N R}\right)\right\rfloor+\left[\left(\frac{1}{N} \times \sum_{i=1}^{N} \frac{1}{N C(\text { w_image, e_watermark })}\right)\right]
$$

1

Where, ' $\gamma$ ' is a stabilizing factor which balances the quality and robustness effects. Algorithm 3 summarizes the complete process of determining the best scaling factor ('opt_sfv') for watermark embedding.

\begin{tabular}{|l|}
\hline Algorithm3: Optimization of scaling factor \\
\hline Input: cover, w_image, PAN, Account \\
Output: opt_sfv \\
begin \\
\hline $1 \mathrm{~T} \leftarrow$ Count (attack); \\
2 [PSNR] $\leftarrow$ embedding_procedure (cover_img, w_image, PAN, Account); \\
3 [NC, BER] $\leftarrow$ Extraction (watermarked_img); \\
\hline 4 for $\mathbf{F} \leftarrow$ Total do \\
\hline 5 [NC[j], BER[j]] $\leftarrow$ extraction_procedure (watermarked_img, attack[j] ); \\
\hline 6 end for \\
\hline 7 fitnessValue $\leftarrow$ objectivefunction ((PSNR, NC, BER), (NC [1], BER [1]), (NC [2], BER [2]) ...... (NC [T], BER [T])); \\
\hline 8 opt_sfv $\leftarrow$ HPSOF (fitnessValue); \\
\hline return opt_sfv \\
\hline
\end{tabular}

Table 3. Regulating parameter for fast convergence 


\begin{tabular}{|ll|}
\hline Regulating Parameter & Value \\
\hline Swarm count & 15 \\
\hline Iteration's cycle & 10 \\
\hline Lower bound (LB) value & 0.005 \\
\hline Upper bound (UB) value & 0.06 \\
\hline Acceleration coefficient1 value & 0.5 \\
\hline Acceleration coefficient2 value & 0.5 \\
\hline Dimensions & 1 \\
\hline employed bees and Onlooker & Swarm count/ 2 \\
\hline Scout bees & Variable \\
\hline
\end{tabular}

\subsection{De-noising process}

DnCNN is employed to enhance the robustness and quality of the recovered watermark. To apply a pre-trained denoising convolutional neural network, the Deep Learning Toolbox is utilised. The denoising procedure for recovered data is summarised in Algorithm 4.

\begin{tabular}{|l|}
\hline Algorithm4: De-noising process \\
\hline Input: e_img \\
Output: e_watermark \\
Begin \\
1 Net=denoisingnewtork (DnCNN) \\
2 e_watermark =denoisingnewtork (e_img, Net) \\
return e_watermark \\
\hline
\end{tabular}

\section{Experiments And Analysis}

In this section, we present the performance of our proposed watermarking algorithm from several perspectives. The experiment was carried out on a 64-bit, $2.50 \mathrm{GHz}$ and 8GB RAM workstation with MATLAB version R2019a. For the validation of the proposed algorithm, the Kodak [28] and USI-SIPI datasets [29] were used and are shown in Figure 2. Each test was performed on colour host images of $512 \times 512$ pixels, user PAN and account details of 80 and 96 bits, respectively, and a grey mark image of $128 \times 128$ pixels. The mark details are presented in Figure 3 . To evaluate and test the proposed algorithm, we have carried out an invisibility analysis using PSNR and SSIM [2], a robustness analysis using NC and BER [2] and a differential analysis using NPCR and UACI [17].

The invisibility, robustness and security performance of the suggested method on the Kodak and USI-SIPI datasets are shown in Table 4. It is noted that the average PSNR value obtained for 40 test images is $57.7124 \mathrm{~dB}$, with the highest value among them being $59.5111 \mathrm{~dB}$. Additionally, the value of NC and BER is approaching 1 and 0 , respectively, in all cases. The average NPCR reaches 0.9956 , and the average UACI score reaches 0.2747 , demonstrating the encryption 
scheme's high security capabilities. Therefore, the results shown in this table are indicative of the suggested method's positive potential. 
Table 4

Performance evaluation on Kodak and USI-SIPI dataset

\begin{tabular}{|c|c|c|c|c|c|c|}
\hline \multirow{2}{*}{$\begin{array}{l}\text { Data set } \\
\text { Kodak dataset (15 test image) }\end{array}$} & \multicolumn{6}{|c|}{ Performance Evaluation } \\
\hline & PSNR & SSIM & NC & BER & NPCR & UACI \\
\hline & 56.7785 & 0.9912 & 1 & 0 & 0.9952 & 0.2789 \\
\hline & 59.3307 & 0.9979 & 1 & 0 & 0.9957 & 0.2795 \\
\hline & 59.0104 & 0.9969 & 1 & 0 & 0.9962 & 0.2692 \\
\hline & 57.8019 & 0.9949 & 1 & 0 & 0.9960 & 0.2703 \\
\hline & 58.0731 & 0.9941 & 1 & 0 & 0.9952 & 0.2719 \\
\hline & 59.5111 & 0.9959 & 1 & 0 & 0.9965 & 0.2786 \\
\hline & 58.5381 & 0.9931 & 1 & 0 & 0.9958 & 0.2707 \\
\hline & 58.0537 & 0.9973 & 1 & 0 & 0.9962 & 0.2701 \\
\hline & 57.1474 & 0.9900 & 1 & 0 & 0.9954 & 0.279 \\
\hline & 58.7135 & 0.9949 & 1 & 0 & 0.9960 & 0.2798 \\
\hline & 59.5084 & 0.9949 & 1 & 0 & 0.9954 & 0.2705 \\
\hline & 58.3774 & 0.9936 & 1 & 0 & 0.9952 & 0.2713 \\
\hline & 57.3104 & 0.9931 & 1 & 0 & 0.9953 & 0.2707 \\
\hline & 58.7151 & 0.9929 & 1 & 0 & 0.9959 & 0.2700 \\
\hline & 58.6988 & 0.9961 & 1 & 0 & 0.9974 & 0.2700 \\
\hline \multirow[t]{15}{*}{ USI-SIPI dataset (25 test image) } & 56.8853 & 0.9909 & 1 & 0 & 0.9943 & 0.2773 \\
\hline & 56.6119 & 0.9930 & 1 & 0 & 0.9954 & 0.2712 \\
\hline & 57.6696 & 0.9964 & 1 & 0 & 0.9954 & 0.2793 \\
\hline & 57.708 & 0.9930 & 1 & 0 & 0.9960 & 0.2692 \\
\hline & 57.9544 & 0.9926 & 1 & 0 & 0.9960 & 0.2794 \\
\hline & 58.2774 & 0.9945 & 1 & 0 & 0.9946 & 0.2782 \\
\hline & 56.0407 & 0.9916 & 1 & 0 & 0.9948 & 0.2794 \\
\hline & 57.5052 & 0.9954 & 1 & 0 & 0.996 & 0.2705 \\
\hline & 56.8698 & 0.9931 & 1 & 0 & 0.9958 & 0.2712 \\
\hline & 57.6771 & 0.9937 & 1 & 0 & 0.9958 & 0.2797 \\
\hline & 56.7700 & 0.9915 & 1 & 0 & 0.9965 & 0.2791 \\
\hline & 57.9248 & 0.9946 & 1 & 0 & 0.9955 & 0.2785 \\
\hline & 58.7132 & 0.9954 & 1 & 0 & 0.9960 & 0.2792 \\
\hline & 57.2670 & 0.9941 & 1 & 0 & 0.9960 & 0.2798 \\
\hline & 56.8517 & 0.9912 & 1 & 0 & 0.9956 & 0.2703 \\
\hline
\end{tabular}




\begin{tabular}{|lllllll|}
\hline Data set & \multicolumn{6}{l|}{ Performance Evaluation } \\
\hline 57.5918 & 0.9937 & 1 & 0 & 0.9958 & 0.2716 \\
\hline 57.2689 & 0.9939 & 1 & 0 & 0.9958 & 0.2700 \\
\hline 56.8311 & 0.9955 & 1 & 0 & 0.9960 & 0.2714 \\
\hline 58.0288 & 0.9935 & 1 & 0 & 0.9961 & 0.2704 \\
\hline 56.7045 & 0.9930 & 1 & 0 & 0.9957 & 0.2793 \\
\hline 57.9820 & 0.9930 & 1 & 0 & 0.9954 & 0.2796 \\
\hline 57.1083 & 0.9927 & 1 & 0 & 0.9953 & 0.2777 \\
\hline 57.9307 & 0.9952 & 1 & 0 & 0.9958 & 0.2787 \\
\hline 55.8526 & 0.9898 & 1 & 0 & 0.9946 & 0.278 \\
\hline Average score of 40 test images & 56.9056 & 0.9907 & 1 & 0 & 0.9949 & 0.2705 \\
\hline
\end{tabular}

Furthermore, to quantify the results, the NC and BER are evaluated for different kinds of attacks, and the average scores are shown in Figure 4 and Table 5, respectively, for 40 test images using the Kodak and USI-SIPI datasets. From Figure 4, it is noted that the satisfactory score of NC, i.e. NC $\geq 0.7863$, is achieved for all considered attacks except the cropping attack. Similarly, the average BER is shown in Table 5 for the same set of dataset images. The average BER score achieved is zero, except for a few attacks (i.e. median filtering, resize and cropping).

Table 5

Average BER score on Kodak and USI-SIPI dataset

\begin{tabular}{|ll|}
\hline Attack & Average BER \\
\hline No attack & 0 \\
\hline Speckle (0.01) & 0 \\
\hline Salt and Pepper (0.005) & 0 \\
\hline Salt and Pepper (0.01) & 0 \\
\hline Median filter [1, 1] & 0 \\
\hline Median filter [2, 2] & 41.6192 \\
\hline Histogram Equalization & 0 \\
\hline Gaussian attack (0.001) & 0 \\
\hline Sharpening Mask (0.1) & 0 \\
\hline JPEG Compression (QF=90) & 0 \\
\hline Resize [512-1024-512] & 0 \\
\hline Resize [512-256-512] & 42.3123 \\
\hline Cropping [20 20 400 480] & 40.4333 \\
\hline
\end{tabular}


PSNR and NC scores are recorded in Table 6 and Figure 5, respectively, and, compared with several other related methods, it can be observed from Table 6 that the achieved PSNR score outperforms the recent methods. Similarly, from Figure 5 , it can be noted that our average NC performs well apart from the cropping attack.

Table 6

Comparison in terms of PSNR

\begin{tabular}{|llllll|}
\hline \multicolumn{2}{|l|}{ PSNR (in dB) } & \multicolumn{3}{l|}{} \\
\begin{tabular}{|l} 
Xu et al. \\
{$[13]$}
\end{tabular} & $\begin{array}{l}\text { Sharma et al. } \\
{[18]}\end{array}$ & $\begin{array}{l}\text { Swaraja et al. } \\
{[30]}\end{array}$ & $\begin{array}{l}\text { Mohan et al. } \\
{[31]}\end{array}$ & $\begin{array}{l}\text { Sinhal et al. } \\
{[32]}\end{array}$ & Average PSNR score \\
& & & & $\begin{array}{l}\text { (Proposed } \\
\text { algorithm) }\end{array}$ \\
39.09 & 47.6391 & 36.99 & 55.1471 & 40.13 & 57.7124 \\
\hline
\end{tabular}

Additionally, to validate the embedding capacity and quality of our proposed method, PSNR scores are recorded in Table 7. It can be observed that the PSNR score of $>51 \mathrm{~dB}$ is achieved after embedding up to 80,000 characters at different gain factor values. The average time cost evaluation for 40 test images is recorded in Table 8 . The average embedding and extraction overhead are 0.177687 and 0.087648 seconds, respectively. Similarly, the average encryption time cost is 0.013705 , and the average decryption time cost is 0.00485 seconds. Table 8 clearly indicates that the execution overhead of the proposed method is acceptable.

Table 7

The PSNR results using different character length

\begin{tabular}{|c|c|c|c|c|c|c|c|c|}
\hline \multirow{2}{*}{$\begin{array}{l}\text { Gain } \\
\text { factor }\end{array}$} & \multicolumn{8}{|c|}{ PSNR (in dB) } \\
\hline & $\begin{array}{l}500 \\
\text { characters }\end{array}$ & $\begin{array}{l}1000 \\
\text { characters }\end{array}$ & $\begin{array}{l}2000 \\
\text { characters }\end{array}$ & $\begin{array}{l}5000 \\
\text { characters }\end{array}$ & $\begin{array}{l}10000 \\
\text { characters }\end{array}$ & $\begin{array}{l}20000 \\
\text { characters }\end{array}$ & $\begin{array}{l}40000 \\
\text { characters }\end{array}$ & $\begin{array}{l}80000 \\
\text { characters }\end{array}$ \\
\hline 0.01 & 57.9955 & 57.908 & 57.751 & 57.3017 & 56.6525 & 55.5715 & 54.0235 & 51.9423 \\
\hline 0.05 & 57.8527 & 57.7616 & 57.6296 & 57.1808 & 56.5522 & 55.5032 & 53.9494 & 51.9106 \\
\hline 0.1 & 57.4713 & 57.3899 & 57.2425 & 56.8392 & 56.2462 & 55.2739 & 53.7900 & 51.8026 \\
\hline 0.06 & 57.7939 & 57.6976 & 57.5643 & 57.1250 & 56.5097 & 55.4612 & 53.9274 & 51.8993 \\
\hline
\end{tabular}

Table 8

Average execution overhead

\begin{tabular}{|ll|}
\hline Process & Time (in seconds) \\
\cline { 2 - 3 } & Cover/Watermark size [512 x 512]/ [128 x 128] \\
\hline Embedding & 0.177687 \\
\hline Extraction & 0.087648 \\
\hline Encryption & 0.013705 \\
\hline Decryption & 0.00485 \\
\hline
\end{tabular}

\section{Conclusions}


A secure colour image watermarking algorithm with improved invisibility, robustness and capacity has been proposed in this paper. In the suggested algorithm, embedding is performed in the spatial and transform domains. Initially, the host colour image is divided into red, green and blue channels. Then, multiple-mark information is concealed in all three channels for different purposes. A trade-off between invisibility and robustness was analysed by fusing PSO and Firefly algorithms. To evaluate the performance of the proposed work, invisibility, robustness and security analyses were performed against various attacks. A detailed comparison was also made, demonstrating how the proposed research is better than the existing algorithms. In the future, we would like to explore the concepts of encryption and compressionbased watermarking, thereby making our system more efficient for other media applications.

\section{Declarations}

Authors' contribution Both authors contributed to the study conception and design. Material preparation, data collection and analysis were performed by both authors. The first draft of the manuscript was written by Dhiran Kumar Mahto and Amit Kumar Singh commented on previous versions of the manuscript. Both authors read and approved the final manuscript.

Funding This work has no funding resource.

\section{Declarations}

Conflict of interest The authors declare that they have no conflict of interest.

Ethical approval This article does not contain any studies with human participants or animals performed by any of the authors.

Consent of publication Not applicable.

\section{References}

1. Sun W, Zhou J, Li Y, Cheung M, She J (2020) Robust High-Capacity Watermarking over Online Social Network Shared Images. IEEE Transactions on Circuits and Systems for Video Technology

2. Mahto DK, Singh AK (2021) A survey of color image watermarking: State-of-the-art and research directions. Computers \& Electrical Engineering, 93, p.107255

3. Urquhart L, McAuley D (2018) Avoiding the internet of insecure industrial things. Computer law \& security review 34(3):450-466

4. Anand A, Singh AK (2020) Watermarking techniques for medical data authentication: a survey. Multimedia Tools and Applications, pp 1-33

5. Singh AK, Kumar B, Singh G, Mohan A (eds) (2017) "Medical image watermarking: techniques and applications". Springer

6. Singh OP, Singh AK, Srivastava G, Kumar N (2020) "Image watermarking using soft computing techniques: A comprehensive survey,"Multimed. Tools Appl.pp.1-32,

7. Cheema AM, Adnan SM, Mehmood Z (2020) A Novel Optimized Semi-Blind Scheme for Color Image Watermarking. IEEE Access 8:169525-169547

8. Pandey MK, Parmar G, Gupta R, Sikander A (2020) Lossless robust color image watermarking using lifting scheme and GWO. International Journal of System Assurance Engineering and Management 11(2):320-331

9. Moosazadeh M, Ekbatanifard G (2019) A new DCT-based robust image watermarking method using teachinglearning-based optimization. Journal of Information Security and Applications 47:28-38

Page $14 / 18$ 
10. Ahmadi SBB, Zhang G, Rabbani M, Boukela L, Jelodar H (2021) An intelligent and blind dual color image watermarking for authentication and copyright protection. Applied Intelligence 51(3):1701-1732

11. Verma VS, Jha RK, Ojha A (2015) Significant region based robust watermarking scheme in lifting wavelet transform domain. Expert Syst Appl 42(21):8184-8197

12. Mohan BC, Swamy KV (2010) On the use of Schur decomposition for copyright protection of digital images. International Journal of Computer and Electrical Engineering 2(4):1793-8163

13. Xu H, Jiang G, Yu M, Luo T (2018) A color image watermarking based on tensor analysis. IEEE Access 6:5150051514

14. Ranjani JJ, Zaid F (2021) Pseudo magic cubes: A multidimensional data hiding scheme exploiting modification directions for large payloads. Comput Electr Eng 89:106928

15. Aydilek IB (2018) A hybrid firefly and particle swarm optimization algorithm for computationally expensive numerical problems. Appl Soft Comput 66:232-249

16. Kumar S, Bhatnagar G (2019) SIE: An application to secure stereo images using encryption. Handbook of Multimedia Information Security: Techniques and Applications. Springer, Cham, pp 37-61

17. Singh OP, Singh AK (2021) Data hiding in encryption-compression domain. Complex \& Intelligent Systems, pp 1-14

18. Sharma S, Sharma H, Sharma JB, Poonia RC (2021) A secure and robust color image watermarking using natureinspired intelligence. Neural Computing and Applications, pp 1-19

19. Su Q, Yuan Z, Liu D (2018) An approximate Schur decomposition-based spatial domain color image watermarking method. IEEE Access 7:4358-4370

20. Sharma S, Sharma H, Sharma JB (2021) Artificial bee colony based perceptually tuned blind color image watermarking in hybrid LWT-DCT domain. Multimedia Tools and Applications 80(12):18753-18785

21. Singh AK (2019) Robust and distortion control dual watermarking in LWT domain using DCT and error correction code for color medical image. Multimedia Tools and Applications 78(21):30523-30533

22. Mohan A, Anand A, Singh AK, Dwivedi R, Kumar B (2021) Selective encryption and optimization-based watermarking for robust transmission of landslide images. Computers \& Electrical Engineering, 95, p.107385

23. Su Q, Chen B (2018) Robust color image watermarking technique in the spatial domain. Soft Comput 22(1):91-106

24. Zear A, Singh PK (2021) Secure and robust color image dual watermarking based on LWT-DCT-SVD. Multimedia Tools and Applications, pp.1-18

25. Kumar S, Singh BK (2021) An improved watermarking scheme for color image using alpha blending. Multimedia Tools and Applications 80(9):13975-13999

26. Loan NA, Hurrah NN, Parah SA, Lee JW, Sheikh JA, Bhat GM (2018) Secure and robust digital image watermarking using coefficient differencing and chaotic encryption. IEEE Access 6:19876-19897

27. Bolourian Haghighi B, Taherinia AH, Monsefi R (2020) An effective semi-fragile watermarking method for image authentication based on lifting wavelet transform and feed-forward neural network. Cognitive Computation 12:863890

28. Kodak dataset at https://www.kaggle.com/sherylmehta/kodak-dataset

29. USI-SIPI dataset http://sipi.usc.edu/database/

30. Swaraja K, Meenakshi K, Kora P (2020) An optimized blind dual medical image watermarking framework for tamper localization and content authentication in secured telemedicine. Biomedical Signal Processing and Control, 55, p.101665

31. Mohan A, Anand A, Singh AK, Dwivedi R, Kumar B (2021) Selective encryption and optimization based watermarking for robust transmission of landslide images. Computers \& Electrical Engineering, 95, p.107385

Page 15/18 
32. Sinhal R, Jain DK, Ansari IA (2021) Machine learning based blind color image watermarking scheme for copyright protection. Pattern Recognit Lett 145:171-177

\section{Figures}

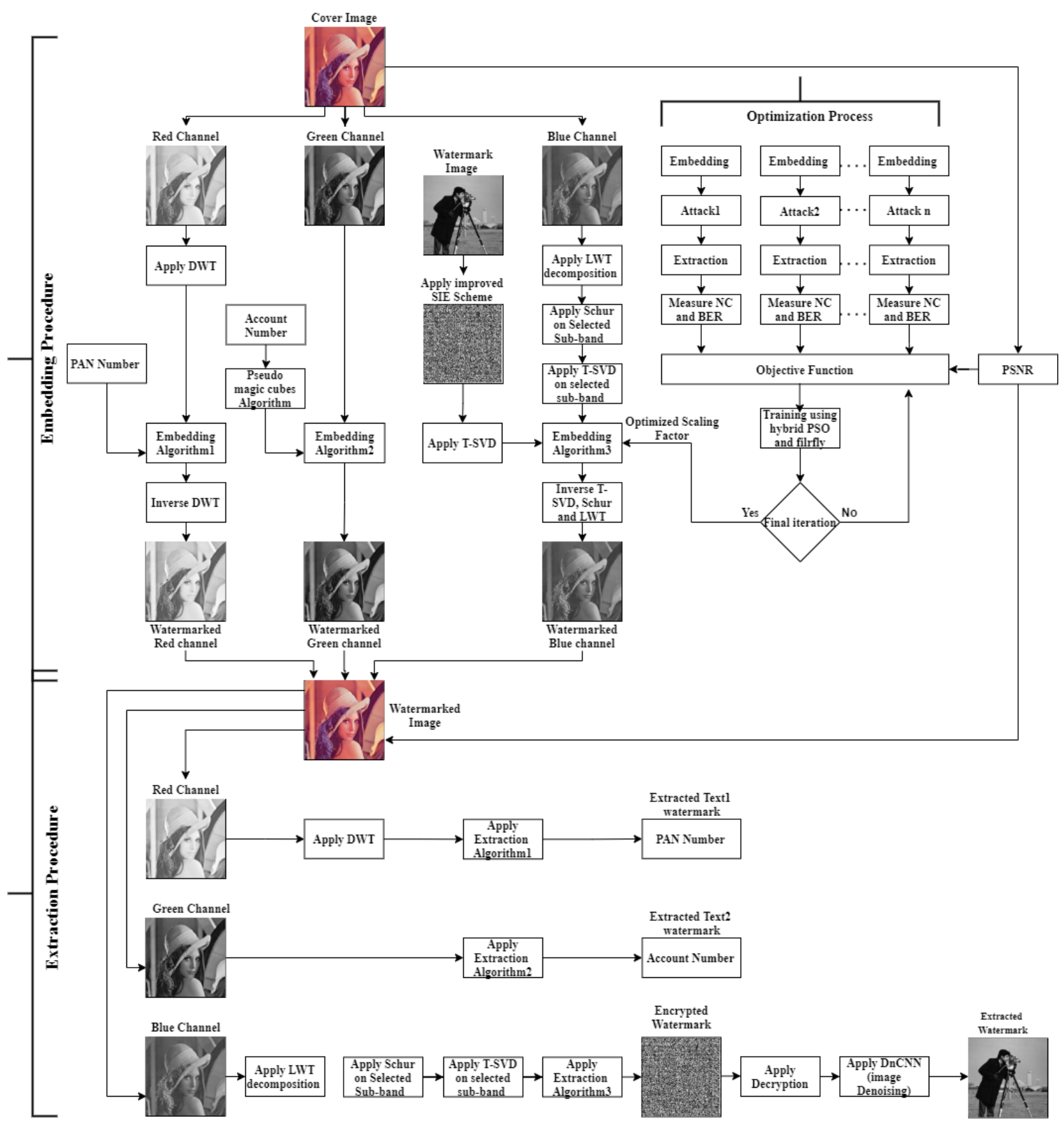

Figure 1

The proposed watermark algorithm 


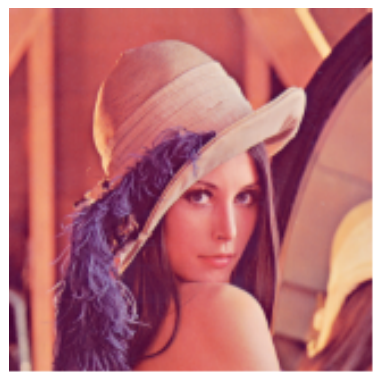

(a) Lena

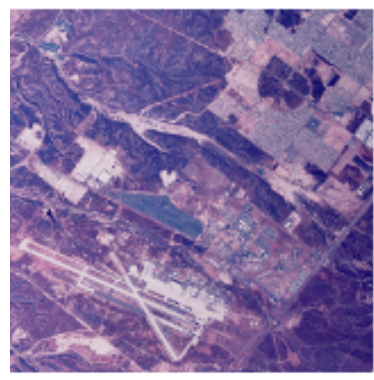

(e) Aeriall

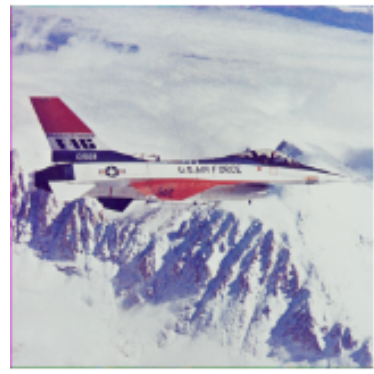

(i) Airplane

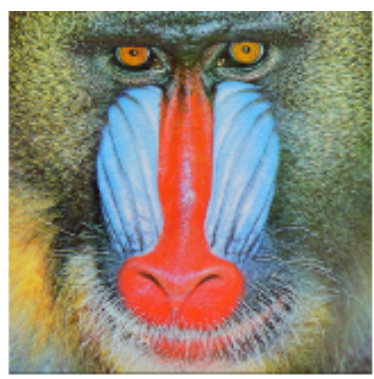

(b) Mandrill

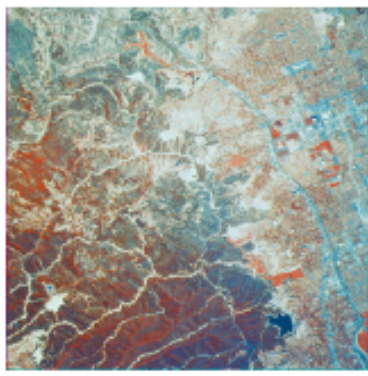

(f) Aerial2

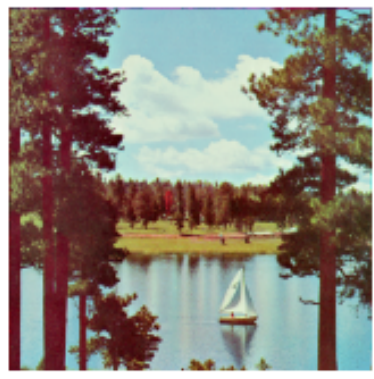

(j) Sailboat

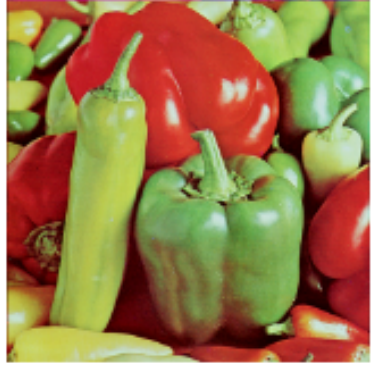

(c) Pepper

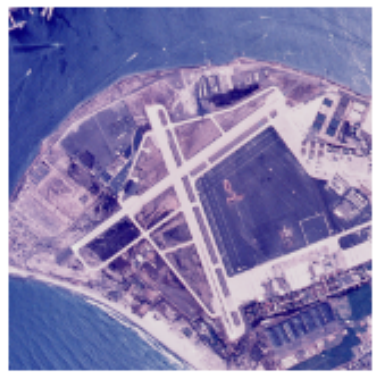

(g) Aerial3

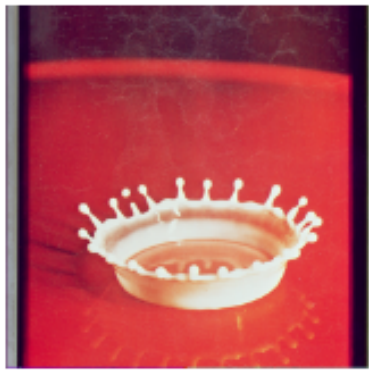

(k) Splash

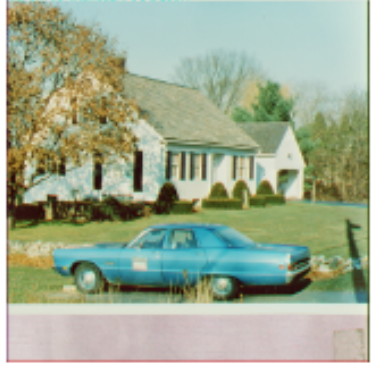

(d) Car

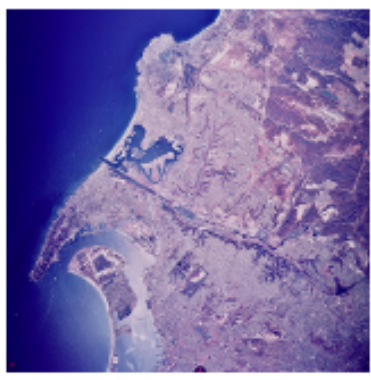

(h) Aerial4

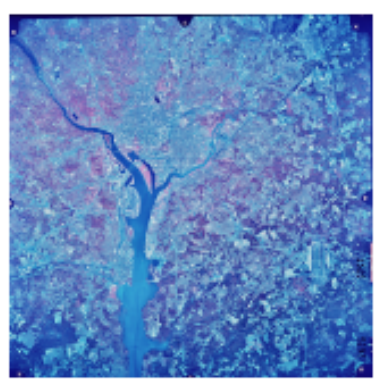

(l) Aerial5

Figure 2

Used some of the test dataset Host images

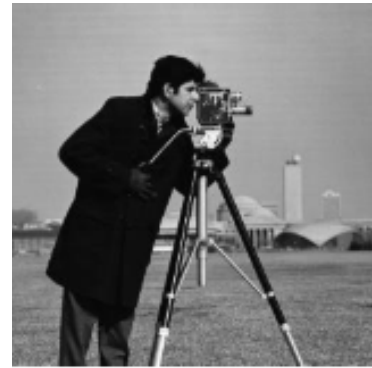

(a) Watermark

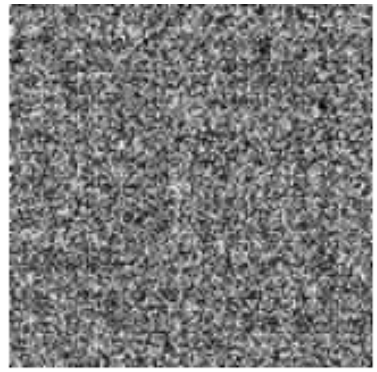

(b) encrypted watermark

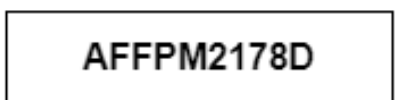

(c) PAN Numbar

\section{7}

(d) Account Number

Figure 3

Used watermarks 


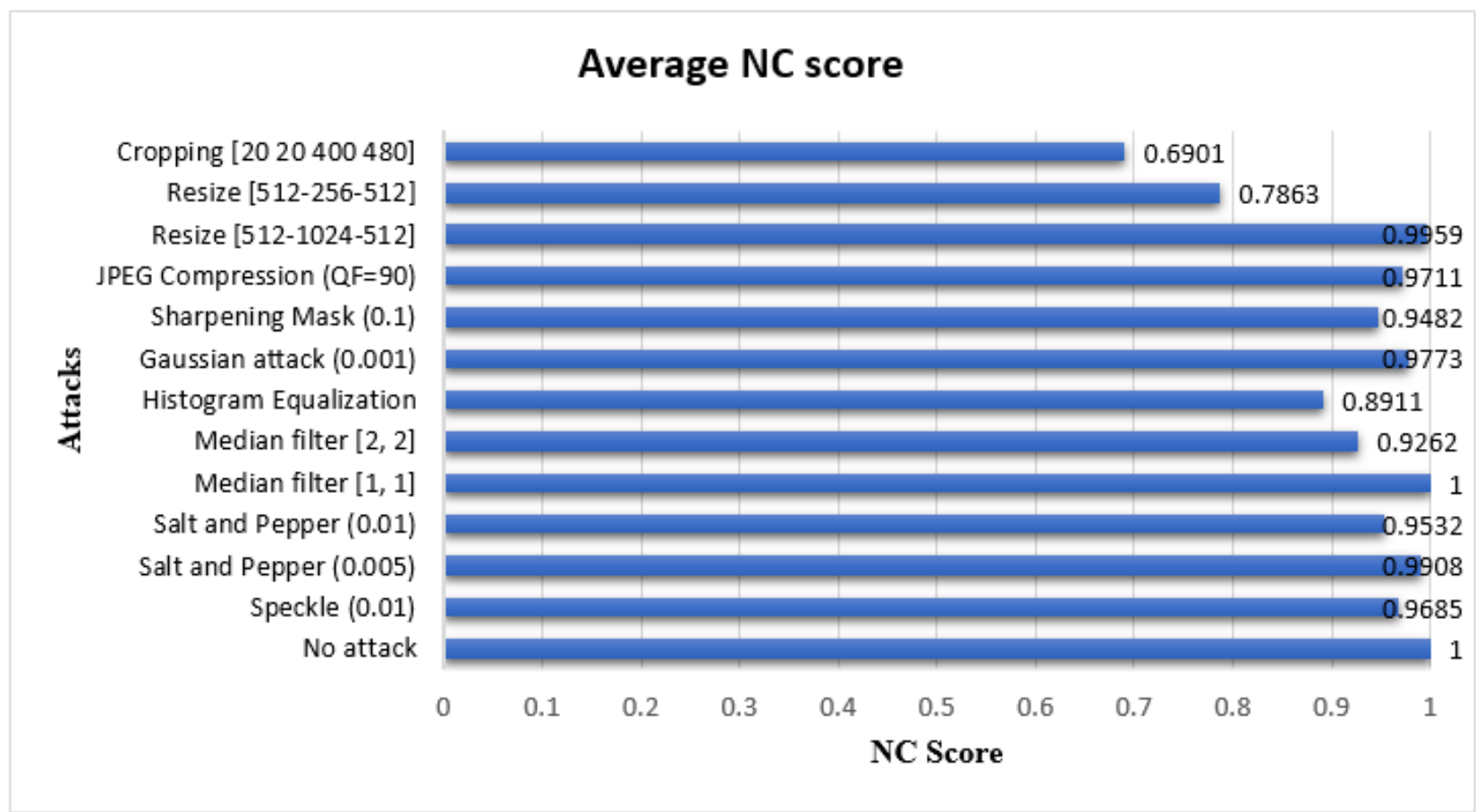

Figure 4

Average NC score on Kodak and USI-SIPI dataset 